\title{
The Faber Polynomials for Circular Sectors
}

\author{
By John P. Coleman and Russell A. Smith
}

\begin{abstract}
The Faber polynomials for a region of the complex plane, which are of interest as a basis for polynomial approximation of analytic functions, are determined by a conformal mapping of the complement of that region to the complement of the unit disc. We derive this conformal mapping for a circular sector $\{z:|z| \leqslant 1,|\arg z| \leqslant \pi / \alpha\}$, where $\alpha>1$, and obtain a recurrence relation for the coefficients of its Laurent expansion about the point at infinity. We discuss the computation of the coefficients of the Faber polynomials of degree 1 to 15 , which are tabulated here for sectors of half-angle $5^{\circ}, 10^{\circ}, 15^{\circ}, 30^{\circ}, 45^{\circ}$, and $90^{\circ}$, and we give explicit expressions, in terms of $\alpha$, for the polynomials of degree $\leqslant 3$. The norms of Faber polynomials are tabulated and are compared with those of the Chebyshev polynomials for the same regions.
\end{abstract}

1. Introduction. In recent years there has been a growing awareness of the potential usefulness of the Faber polynomials as a basis for polynomial and rational approximations in the complex plane. It has been shown [4], [3] that, under mild conditions, a truncated Faber series is a near-minimax polynomial approximation for an analytic function, on the region to which it applies. However, practical exploitation of this result has been hampered by a lack of knowledge of Faber polynomials, except for a few specific regions. In particular, for circular sectors, which provide a convenient subdivision of the plane, the Faber polynomials were hitherto unknown.

For any closed bounded continuum $D$ in the complex plane there is a function $\phi$, such that

$$
\lim _{z \rightarrow \infty} \frac{\phi(z)}{z}=1
$$

which maps the complement of $D$ in the extended $z$-plane conformally onto $\{w$ : $|w|>\rho\}$, the complement of a closed disc of radius $\rho$ (see, e.g., Markushevich [10, v. 3 , p. 104]). The number $\rho$ is called the transfinite diameter of $D$. The function $\phi$ has a Laurent expansion

$$
\phi(z)=z+\alpha_{0}+\frac{\alpha_{1}}{z}+\cdots
$$

about the point at infinity. The Faber polynomial of degree $n$ is obtained by deleting all negative powers of $z$ from the corresponding Laurent expansion of $[\phi(z)]^{n}$. For the unit disc the Faber polynomial of degree $n$ is $z^{n}$ and the corresponding Faber series for an analytic function is its Taylor series about the origin. Multiples of the

Received February 27, 1986; revised July 23, 1986.

1980 Mathematics Subject Classification (1985 Revision). Primary 30C10, 30C20, 30E10. 
Chebyshev polynomials are the Faber polynomials for an ellipse with foci at $( \pm 1,0)$, and, in particular, for the real interval $[-1,1]$.

Properties of the Faber polynomials and Faber series are described in the books of Markushevich [10, v. 3, pp. 104-112], Smirnov and Lebedev [12, Chapter 2] and Gaier [5, pp. 46-57], and in a survey article by Curtiss [2]. Markushevich [10] shows how Faber polynomials may be obtained for certain lemniscates, and Elliott [4] computed the coefficients of some Faber polynomials for the semidisc $|z| \leqslant 1$, $\operatorname{Re} z \geqslant 0$ and for the square $|\operatorname{Re} z| \leqslant 1,|\operatorname{Im} z| \leqslant 1$. Ellacott [3] pointed out that the trapezium rule could be used to compute the coefficients of Faber polynomials when the mapping function $\phi$ or its inverse is known. Thus the difficulty, for any particular region, lies in finding the appropriate conformal mapping.

The main theoretical results of this paper are summarized in the following three theorems.

THEOREM 1. The complement of the unit disc $\{w:|w| \leqslant 1\}$ is mapped conformally onto the complement of the circular sector $\{z:|z| \leqslant 1,|\arg z| \leqslant \pi / \alpha\}$, with $\alpha>1$, by

$$
\psi(w)=\frac{-\left[u+\left(u^{2}-1\right)^{1 / 2}\right]^{2}}{\left[v+\left(v^{2}-1\right)^{1 / 2}\right]^{p(a)}},
$$

where

$$
u(w)=\frac{i(w-1)}{2 a w^{1 / 2}}, \quad v(w)=\frac{\left(2-a^{2}\right)(w-1)^{2}+4 w a^{2}}{a^{2}(w+1)^{2}}
$$

with $a=\alpha^{-1}(2 \alpha-1)^{1 / 2}$ and $p(a)=\left(1-a^{2}\right)^{1 / 2}$.

THEOREM 2. The transfinite diameter of the sector $\{z:|z| \leqslant 1,|\arg z| \leqslant \pi / \alpha\}$, with $\alpha \geqslant 1$, is

$$
\rho=\frac{\alpha^{2}}{(2 \alpha-1)^{2-1 / \alpha}} .
$$

THEOREM 3. The coefficients of the Laurent expansion

$$
\psi(w)=\rho\left(w+\beta_{0}+\beta_{1} w^{-1}+\cdots\right)
$$

of the function defined in Theorem 1 may be generated recursively. Given $\alpha$ and $p$ as in the statement of Theorem 1 , let $x=2 p^{2}-1, a_{0}=1$ and, for $k \geqslant 1$,

$$
a_{k}=P_{k}(x)+P_{k-1}(x),
$$

where $P_{n}(x)$ is the Legendre polynomial of degree $n$. Then $\beta_{0}=a_{1}$ and, for $k \geqslant 1$,

$$
(k+1) \beta_{k}=a_{k+1}-\sum_{\nu=0}^{k-1} \nu \beta_{\nu} a_{k-\nu} .
$$

Theorems 1 and 2 are proved in Section 2, and Theorem 3 in Section 3. The numerical computation of coefficients of Faber polynomials for circular sectors is described in detail in Section 4, and in Section 5 the relationship of the norms of Faber and Chebyshev polynomials for circular sectors is discussed. Tables of coefficients of Faber polynomials, of degree up to 15 , for selected sectors can be found in the Supplements section of this issue. 
2. The Conformal Mapping. In this section we aim to calculate an analytic function $\psi(w)$ which maps the complement of the unit disc $\Delta=\{w:|w| \leqslant 1\}$ conformally onto the complement of the circular sector $S_{h}=\{z:|z| \leqslant 1,|\arg z| \leqslant$ $2 h\}$, where $0<h<\frac{1}{2} \pi$. Let $J$ denote the interval $[-1,1]$ of the real axis. Then the function $\frac{1}{2}\left(w^{-1}+w\right)$ maps $\mathbf{C}-\Delta$ conformally onto the slit plane $\mathbf{C}-J$ (see [8, p. 59]). If $\psi_{0}(z)$ is a function which maps $\mathbf{C}-J$ conformally onto $\mathbf{C}-S_{h}$, then $\psi(w)=\psi_{0}\left(\frac{1}{2}\left(w^{-1}+w\right)\right)$ is the required function. It is therefore sufficient to calculate $\psi_{0}(z)$.

Let $K$ denote the interval $(-\infty, 1]$ of the real axis. Then the cut plane $\mathbf{C}-K$ is mapped by the function $2^{-1 / 2} i(z-1)^{1 / 2}$ conformally onto the upper half-plane $\Pi=\{z: \operatorname{Im} z>0\}$. A Schwarz-Christoffel transformation $\sigma(z)$ can be used to map $\Pi$ conformally onto the shaded domain $E$ shown in Figure 1 . In [8, p. 160] this mapping is given by

$$
\sigma(z)=\frac{i \pi}{2}-\cosh ^{-1} \frac{z}{a}+\frac{\left(1-a^{2}\right)^{1 / 2}}{2} \cosh ^{-1} \frac{\left(2-a^{2}\right) z^{2}-a^{2}}{a^{2}\left(z^{2}-1\right)},
$$

where $0<a<1$. The constant $h$ in Figure 1 is given by

$$
a=\pi^{-1} 2\left(\pi h-h^{2}\right)^{1 / 2}, \quad h=\frac{1}{2} \pi\left[1-\left(1-a^{2}\right)^{1 / 2}\right] .
$$

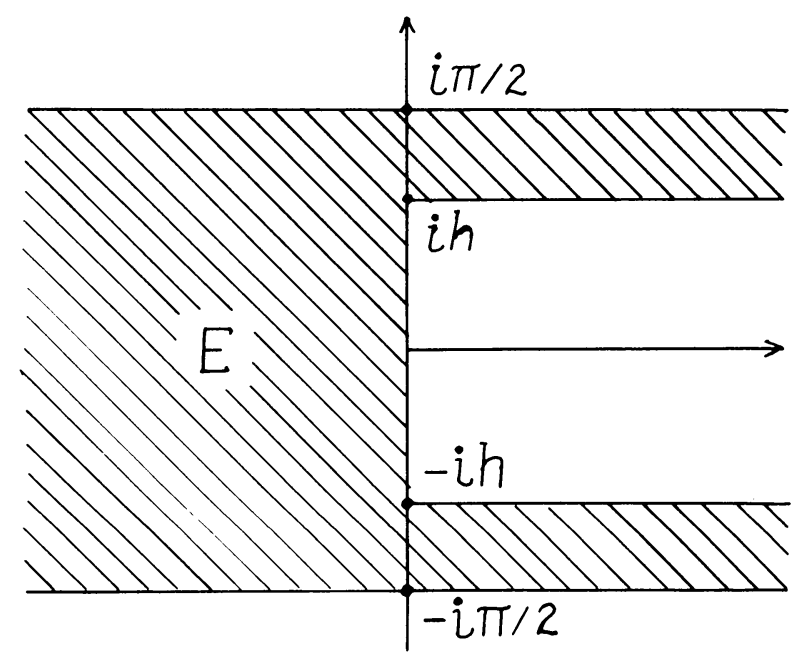

Figure 1

The Schwarz-Christoffel transformation $\sigma(z)$ given by Eq.

(2) maps the upper half-plane conformally onto the shaded region $E$ shown here.

Finally, the domain $E$ is mapped by the function $e^{-2 z}$ conformally onto the domain obtained by cutting $\mathrm{C}-S_{h}$ along the negative real axis. The upper and lower boundary lines of $E$ are both mapped by $e^{-2 z}$ onto the cut. The composition function $\psi_{0}(z)=\exp \left[-2 \sigma\left(2^{-1 / 2} i(z-1)^{1 / 2}\right)\right]$ therefore maps $\mathbf{C}-K$ conformally onto this cut version of $\mathbf{C}-S_{h}$.

Since $\cosh ^{-1} \theta=\log \left[\theta+\left(\theta^{2}-1\right)^{1 / 2}\right],(2)$ can be written as

$$
\sigma(z)=\frac{i \pi}{2}-\log \frac{z+\left(z^{2}-a^{2}\right)^{1 / 2}}{a}+\frac{p(a)}{2} \log \frac{\left[\left(z^{2}-a^{2}\right)^{1 / 2}+z p(a)\right]^{2}}{a^{2}\left(z^{2}-1\right)},
$$


where $p(a)=\left(1-a^{2}\right)^{1 / 2}$. This gives $\psi_{0}(z)=U(z) V(z)^{p(a)}$, where

$$
\begin{aligned}
& U(z)=\frac{1}{2} a^{-2}\left[(z-1)^{1 / 2}+\left(z-1+2 a^{2}\right)^{1 / 2}\right]^{2}, \\
& V(z)=(z+1) a^{2}\left[\left(z-1+2 a^{2}\right)^{1 / 2}+(z-1)^{1 / 2} p(a)\right]^{-2} .
\end{aligned}
$$

It is easily seen that, in the slit plane $\mathbf{C}-J$, the functions $U(z), V(z)$ are analytic and single-valued with $V(z) \neq 0$. Then $V(z)^{p(a)}$ is analytic in $\mathbf{C}-J$, though it might not be single-valued because $\mathbf{C}-J$ is not simply-connected. However, $V(z)^{p(a)}$ can only have branch points where $V(z)=0$, and this does not happen at $\infty$ because (5) shows that $V(z) \rightarrow a^{2}[1+p(a)]^{-2}$ as $z \rightarrow \infty$. Hence $V(z)^{p(a)}$ is single-valued in some neighborhood of $\infty$ and therefore single-valued in the whole of $\mathbf{C}-J$ by the monodromy theorem (see [10, v. 3, p. 269]). Thus $\psi_{0}(z)$ is analytic and single-valued in $\mathbf{C}-J$. It is easily seen that the part of $K$ which lies in $\mathbf{C}-J$ is mapped by $\psi_{0}(z)$ onto the whole of the negative real axis. That is, $\psi_{0}(z)$ maps C $-J$ conformally onto $\mathrm{C}-S_{h}$.

As shown above, $\mathbf{C}-\Delta$ is mapped conformally onto $\mathbf{C}-S_{h}$ by

$$
\psi(w)=\psi_{0}\left(\frac{1}{2}\left(w^{-1}+w\right)\right)=U\left(\frac{1}{2}\left(w^{-1}+w\right)\right) V\left(\frac{1}{2}\left(w^{-1}+w\right)\right)^{p(a)} .
$$

For computational convenience this can be written as

$$
\psi(w)=\frac{-\left[u+\left(u^{2}-1\right)^{1 / 2}\right]^{2}}{\left[v+\left(v^{2}-1\right)^{1 / 2}\right]^{p(a)}},
$$

where

$$
u(w)=\frac{i(w-1)}{2 a w^{1 / 2}}, \quad v(w)=\frac{\left(2-a^{2}\right)(w-1)^{2}+4 w a^{2}}{a^{2}(w+1)^{2}} .
$$

The appropriate branch for the square root $\left(u^{2}-1\right)^{1 / 2}$ is that for which

$$
u^{-1}\left(u^{2}-1\right)^{1 / 2} \rightarrow 1 \text { as } u \rightarrow+\infty .
$$

Since (4) gives $z^{-1} U(z) \rightarrow 2 a^{-2}$ as $z \rightarrow \infty$, it follows that

$$
\lim _{x \rightarrow \infty} \frac{\psi(w)}{w}=\lim _{z \rightarrow \infty} \frac{\psi_{0}(z)}{2 z}=a^{-2}\left[\frac{a}{1+p(a)}\right]^{2 p(a)}=\rho .
$$

Now define $\phi(z)=\rho \psi^{-1}(z)$ where $w=\psi^{-1}(z)$ denotes the inverse mapping of $z=\psi(w)$. Then $\phi(z)$ maps $\mathbf{C}-S_{h}$ conformally onto the complement of the disc $\{w:|w| \leqslant \rho\}$. The number $\rho$ is therefore the transfinite diameter of the sector $S_{h}$ because (8) ensures that $\phi(z)$ satisfies (1). If the angle of the sector $S_{h}$ is denoted by $2 \pi / \alpha$, then $1<\alpha=\pi / 2 h$ and (3) gives $a=\alpha^{-1}(2 \alpha-1)^{1 / 2}$. Then $p(a)=1-\alpha^{-1}$, and (8) gives

$$
\rho=\frac{\alpha^{2}}{(2 \alpha-1)^{2-1 / \alpha}} .
$$

Some checks which confirm the accuracy of (6) and (9) will now be discussed. When $\alpha \rightarrow 1$, (6) gives $\psi(w) \rightarrow w$ and (9) gives $\rho \rightarrow 1$. These are the expected limits because $S_{h} \rightarrow \Delta$ as $\alpha \rightarrow 1$. When $\alpha \rightarrow+\infty,(6)$ gives $\psi(w) \rightarrow(w+1)^{2} / 4 w$ and (9) gives $\rho \rightarrow 1 / 4$. These are the expected limits because $S_{h}$ tends to the interval $[0,1]$ of 
the real axis when $\alpha \rightarrow+\infty$. When $\alpha=2$, (9) gives $\rho=4 / 3 \sqrt{3}$ and (6) gives

$$
\begin{aligned}
\psi(w) & =-\left[u+\left(u^{2}-1\right)^{1 / 2}\right]^{2}\left[v-\left(v^{2}-1\right)^{1 / 2}\right]^{1 / 2} \\
& =\frac{\left[w-1+\left(w^{2}+w+1\right)^{1 / 2}\right]^{2}\left[1-w+2\left(w^{2}+w+1\right)^{1 / 2}\right]}{w(w+1) 3 \sqrt{3}} \\
& =\frac{2\left(w^{3}-1\right)+3\left(w^{2}-w\right)+2\left(w^{2}+w+1\right)^{3 / 2}}{w(w+1) 3 \sqrt{3}} .
\end{aligned}
$$

In this case, $S_{h}$ is the unit semidisc for which Elliott [4] obtained the transfinite diameter $\rho=4 / 3 \sqrt{3}$ by using a conformal mapping for which the appropriate inverse is

$$
\psi_{e}(w)=i\left(b^{3 / 2}+c^{3 / 2}\right) /\left(b^{3 / 2}-c^{3 / 2}\right),
$$

where $b=w+e^{i \pi / 3}, c=w+e^{-i \pi / 3}$. This can be written as

$$
\psi_{e}(w)=\frac{i\left(b^{3 / 2}+c^{3 / 2}\right)^{2}}{b^{3}-c^{3}}=\frac{i\left[b^{3}+c^{3}+2(b c)^{3 / 2}\right]}{b^{3}-c^{3}} .
$$

Since $b^{3}-c^{3}=i w(w+1) 3 \sqrt{3}, \quad b c=1+w+w^{2}$ and $b^{3}+c^{3}=2\left(w^{3}-1\right)+$ $3\left(w^{2}-w\right)$, this and (10) give $\psi_{e}(w)=\psi(w)$. That is, Elliott [4] agrees with the above calculations.

3. The Laurent Expansion of $\psi(w)$ About the Point at Infinity. The function $\psi(w)$, given by Eq. (6), has the Laurent expansion

$$
\psi(w)=\rho\left(w+\beta_{0}+\beta_{1} w^{-1}+\cdots\right)
$$

about the point at infinity. The presence of the coefficients of this expansion in a recurrence relation for the Faber polynomials (Eq. (17) below) provides the motivation for the derivation of a recurrence relation from which $\left\{\beta_{k}\right\}$ may be calculated.

Differentiation of (6) and (7) gives

$$
\frac{d \psi}{d w}=\psi(w)\left[\frac{2 u^{\prime}}{\sqrt{u^{2}-1}}-\frac{p v^{\prime}}{\sqrt{v^{2}-1}}\right]
$$

with

$$
u^{\prime}=\frac{i(1+w)}{4 a w \sqrt{w}}, \quad v^{\prime}=\frac{8 p^{2}(w-1)}{a^{2}(w+1)^{3}}
$$

and, since

$$
\sqrt{v^{2}-1}=\frac{2 p u \sqrt{u^{2}-1}}{a^{2} u^{2}-1}
$$

this finally reduces to

$$
\frac{d \psi}{d w}=\frac{\psi(w)}{w}\left[1-\frac{4 p^{2} w}{(w+1)^{2}}\right]^{1 / 2} .
$$

It is now convenient to let $\zeta=w^{-1}$ and $\Psi(\zeta)=\psi(w)$, so that (12) becomes

$$
\Psi(\zeta)=-\left(1-2 x \zeta+\zeta^{2}\right)^{-1 / 2}(1+\zeta) \zeta \frac{d \Psi}{d \zeta}
$$


where $x=2 p^{2}-1$. Then $-1<x<1$ because $p=1-\alpha^{-1}$ and $\alpha>1$. For $|\zeta|<1$,

$$
\left(1-2 x \zeta+\zeta^{2}\right)^{-1 / 2}=\sum_{n=0}^{\infty} P_{n}(x) \zeta^{n},
$$

where $P_{n}(x)$ is the Legendre polynomial of degree $n$, and (13) gives

$$
\Psi(\zeta)=\left[\sum_{k=0}^{\infty} a_{k} \zeta^{k}\right]\left[-\zeta \frac{d \Psi}{d \zeta}\right]
$$

where $a_{0}=1$ and, for $k \geqslant 1$,

$$
a_{k}=P_{k}(x)+P_{k-1}(x) .
$$

From (11),

$$
\Psi(\zeta)=\rho\left[\zeta^{-1}+\sum_{\nu=0}^{\infty} \beta_{\nu} \zeta^{\nu}\right]
$$

for $|\zeta|<1$. By substituting this in (14) and equating the coefficients of $\zeta^{k}$ on both sides, we find that $\beta_{0}=a_{1}$ and, for $k \geqslant 1$,

$$
(k+1) \beta_{k}=a_{k+1}-\sum_{\nu=0}^{k-1} \nu \beta_{\nu} a_{k-\nu} .
$$

The Legendre polynomials, and hence the coefficients $a_{k}$, may be generated by using the recurrence relation

$$
(n+1) P_{n+1}(x)-(2 n+1) x P_{n}(x)+n P_{n-1}(x)=0 .
$$

4. Computational Details. The Faber polynomials $\phi_{n}$ satisfy the recurrence relation

$$
\phi_{n+1}(z)=z \phi_{n}(z)-\sum_{k=0}^{n-1} b_{k} \phi_{n-k}(z)-(1+n) b_{n}, \quad n>0
$$

(see, e.g., Ellacott [3]), with $b_{k}=\beta_{k} \rho^{k+1}$, where $\rho$ is the transfinite diameter of the region of interest and $\beta_{k}$ is a coefficient of the Laurent expansion (11). In particular, for a sector of half-angle $\pi / \alpha$, Eq. (15) and the recurrence relation (16) give

$$
\begin{gathered}
a_{1}=1+x, \quad a_{2}=\frac{1}{2}(1+x)(3 x-1), \quad a_{3}=\frac{1}{2}(1+x)\left(5 x^{2}-2 x-1\right), \\
b_{0}=\rho(1+x), \quad b_{1}=\rho^{2}(1+x)(3 x-1) / 4, \\
b_{2}=-\rho^{3}\left(1-x^{2}\right)(7 x+1) / 12
\end{gathered}
$$

with $x=1-2 a^{2}$ and $a=\alpha^{-1}(2 \alpha-1)^{1 / 2}$. It follows that the Faber polynomials of degree $\leqslant 3$ are

$$
\begin{aligned}
& \phi_{0}(z)=1 \\
& \phi_{1}(z)=z-2 \rho\left(1-a^{2}\right) \\
& \phi_{2}(z)=z^{2}-4 \rho\left(1-a^{2}\right) z+2 \rho^{2}\left(1-a^{4}\right),
\end{aligned}
$$

and

$$
\begin{aligned}
\phi_{3}(z)= & z^{3}-6 \rho\left(1-a^{2}\right) z^{2}+3 \rho^{2}\left(1-a^{2}\right)\left(3-a^{2}\right) z \\
& -2 \rho^{3}\left(1-a^{2}\right)\left(2 a^{4}+1\right) .
\end{aligned}
$$

Faber polynomials of higher degree could also be generated in this way but we chose to base our computations on an alternative numerical method which is more versatile and permits independent checks on its accuracy. 
The coefficients of a Faber polynomial of degree $n$,

$$
\phi_{n}(z)=\sum_{k=0}^{n} C_{k}^{(n)} z^{k},
$$

may be expressed in a number of ways in terms of the relevant mapping (see, e.g., Ellacott [3]). In particular,

$$
C_{k}^{(n)}=\frac{\rho^{n}}{2 \pi i} \oint_{|w|=R} \frac{w^{n}(d \psi / d w)}{[\psi(w)]^{k+1}} d w
$$

for any $R>1$. This differs by a factor of $\rho^{n}$ from the expression quoted by Ellacott [3] because in his notation $\psi$ maps the complement of the disc $\{w:|w| \leqslant \rho\}$, rather than that of the unit disc, onto the region of interest in the $z$-plane.

Equation (12) gives the derivative of $\psi$ in a convenient form and, when this is used, Eq. (20) becomes

$$
C_{k}^{(n)}=\frac{(R \rho)^{n}}{2 \pi} \int_{0}^{2 \pi} F_{k}\left(R e^{i \theta}\right) \exp (\operatorname{in} \theta) d \theta
$$

where

$$
F_{k}(w)=\left[1-\frac{4 p^{2} w}{(w+1)^{2}}\right]^{1 / 2} /[\psi(w)]^{k} .
$$

Since the integrand in (21) is a periodic function which is integrated over a period, $C_{k}^{(n)}$ may be computed efficiently by the trapezium rule, which gives

$$
C_{k}^{(n)} \simeq \frac{(R \rho)^{n}}{N} \sum_{m=0}^{N-1} F_{k}\left(R \exp \left(i \theta_{m}\right)\right) \exp \left(i n \theta_{m}\right)
$$

with $\theta_{m}=2 \pi m / N$, when $N$ is the number of equal subintervals used.

For a given value of $n$ the coefficients $C_{k}^{(n)}$, for $k=0,1, \ldots, n$, may be calculated from (23) using a suitable value of $N$, but this is inefficient if more than one Faber polynomial is to be found. The values of $w$ used in (22) depend on the choice of $R$ and $N$ but not on $n$. When $R$ and $N$ are chosen, the calculation of the coefficients $C_{k}^{(n)}$, for Faber polynomials of degree $n_{\min }$ to $n_{\max }$, may be arranged as follows.

1. Preliminary calculation.

For $m=0,1, \ldots, N-1$ :

Calculate $w_{m}=R \exp \left(i \theta_{m}\right)$.

Calculate and store $\psi\left(w_{m}\right)$ and the numerator of the expression in (22), with $w=w_{m}$.

2. Computation of coefficients.

For $k=0,1, \ldots, n$ :

2.1. For $m=0,1, \ldots, N-1$ :

Calculate $F_{k}\left(w_{m}\right)$ from stored data.

2.2. For $n=n_{\min }, \ldots, n_{\max }$ :

Calculate $C_{k}^{(n)}$ from (23).

This arrangement, in which $C_{k}^{(n)}$ is calculated for a given $k$ and all relevant $n$, would allow the use of a Fast Fourier Transform algorithm for the step 2.2, as suggested by Lyness and Sande [9] for the evaluation of Taylor coefficients, and by Ellacott [3] in connection with another formula for the coefficients of Faber polynomials. 
Properties of the Faber polynomials provide a number of ways of assessing the accuracy of computed coefficients.

(i) By definition, the Faber polynomials are monic, so $C_{n}^{(n)}=1$ for each $n$. Computation of these coefficients provides a particularly valuable check, as it turns out that for any $n$ the coefficient most sensitive to discretization error is $C_{n}^{(n)}$; Eq. (22) shows that this is to be expected since a higher power of $z=\psi(w)$ occurs in $F_{n}(w)$ than in $F_{k}(w)$ for $k<n$.

(ii) It is readily seen that $C_{n-1}^{(n)}=-n b_{0}$, and the recurrence relation (17) gives

$$
C_{n-2}^{(n)}=C_{n-3}^{(n-1)}-b_{0} C_{n-2}^{(n-1)}-b_{1}
$$

and

$$
C_{n-3}^{(n)}=C_{n-4}^{(n-1)}-b_{0} C_{n-3}^{(n-1)}-b_{1} C_{n-3}^{(n-2)}-b_{2} .
$$

Thus, knowing $b_{0}, b_{1}$ and $b_{2}$ from (18) we have an alternative method of evaluating the coefficients of $z^{n-1}, z^{n-2}$ and $z^{n-3}$ in $\phi_{n}(z)$. In particular, from the equations (19), the polynomials $\phi_{1}(z), \phi_{2}(z)$ and $\phi_{3}(z)$ are known for any sector.

(iii) For a sector of half-angle $\pi / \alpha$, with $\alpha$ rational, $a^{2}$ is a rational number and, therefore, so also are the coefficients $\beta_{0}, \beta_{1}, \ldots$ of the Laurent expansion (11). It follows from the recurrence relation (17) that $C_{k}^{(n)} \rho^{k-n}$ is a rational number. Tabulation of this quantity provides a valuable visual check, particularly for $\alpha=2$ or 4.

(iv) The coefficients $C_{k}^{(n)}$ are real but, since the sum in (23) involves complex quantities, the imaginary part of the computed result will not be exactly zero. However, this does not provide a useful check as the size of the imaginary part obtained gives no indication of the magnitude of the discretization error in the real part.

Although in principle any $R>1$ may be used in (23), the choice of $R$ was found to have a very significant effect on the accuracy of the results obtained for a given $N$, the number of terms in the sum. A study of the results of our computations in double- and quadruple-precision leads to the following conclusions.

(a) The discretization error is dramatically decreased by increasing $N$ or $R$. With $R=3$ and $N=128$, the coefficients of the Faber polynomials of degree $\leqslant 15$, for the sectors investigated, were correct to all 25 digits printed by the quadruple-precision program. However, for a sector of half-angle $5^{\circ}$, with $N=128$, the calculated value of the unit coefficient $C_{15}^{(15)}$ is in error by $1.4 \times 10^{-7}$ when $R=2$, and calculations with $R=1.5$ return the ridiculous value $1.4 \times 10^{9}$ instead of 1 . For the same sector, with $R=3$, the computed value of $C_{15}^{(15)}$ is in error by $3.9 \times 10^{-7}$ when $N=64$, and is correct to more than 25 digits when $N=128$, as already mentioned, but becomes $1.4 \times 10^{3}$ when $N=32$; thus a four-fold increase in $N$ reduces the error in this case by more than 28 orders of magnitude.

(b) For any given sector, and given values of $N$ and $R$, the discretization error increases with the degree of the polynomial, and for a polynomial of degree $n$ it is greatest for the unit coefficient $C_{n}^{(n)}$. For example, when $R=3$ and $N=32$, although $C_{15}^{(15)}$ for the sector of half-angle $5^{\circ}$ is returned as $1.4 \times 10^{3}$, the error in the calculated value of $C_{0}^{(15)}$ is merely $9 \times 10^{-24}$, making that coefficient correct to 15 significant digits; the corresponding errors in the unit coefficients $C_{10}^{(10)}, C_{5}^{(5)}$ and $C_{1}^{(1)}$ are $4.9 \times 10^{-2}, 7.9 \times 10^{-7}$, and $4.8 \times 10^{-13}$, respectively. 
(c) The discretization error increases, for given values of $N$ and $R$, as the angle of the sector is decreased. When $N=64$ and $R=3$, for example, the discretization errors in the computed values of $C_{15}^{(15)}$ for sectors of half-angle $5^{\circ}, 10^{\circ}, 30^{\circ}$ and $90^{\circ}$ are, respectively, $3.9 \times 10^{-7}, 9.4 \times 10^{-8}, 2.9 \times 10^{-10}$, and $2.3 \times 10^{-18}$.

(d) As $R$ is increased, the image, under the mapping $\psi$, of the circle $|w|=R$ becomes more nearly circular. As this happens, rounding error becomes increasingly apparent in double-precision calculations, and it was evidence of this rounding error which prompted the quadruple-precision calculations. For any given sector the rounding error increases with increasing $R$, and for any given $R$ it is greater for the unit semidisc than for any smaller sector, which is consistent with the fact that the images of $|w|=R$ become nearly circular more rapidly for the unit semidisc than for smaller sectors. For example, for the unit semidisc, the rounding error in the value of $C_{0}^{(15)}$, calculated in double-precision with $N=128$, is $2.5 \times 10^{-10}$ when $R=4$ and $1.0 \times 10^{-14}$ when $R=2$; the corresponding errors in $C_{0}^{(15)}$ for a sector of half-angle $30^{\circ}$ are $2.2 \times 10^{-14}$ and $1.0 \times 10^{-17}$, respectively.

5. The Faber Polynomials for Circular Sectors. A supplement to this paper contains the coefficients of the Faber polynomials, of degree 1 to 15 , for symmetric sectors of the unit disc of half-angle $5^{\circ}, 10^{\circ}, 15^{\circ}, 30^{\circ}, 45^{\circ}$ and $90^{\circ}$. The polynomials for sectors of radius other than 1 , and for angular ranges of the form $\theta \leqslant \arg z \leqslant \phi$, may be obtained, if required, by simple changes of variable. The tabulated coefficients are believed to be correct to the number of figures quoted, having been obtained by rounding to 14 decimal places results which all our tests show to be accurate to 25 decimal digits. Comparison with previous work is possible only in the case of the semidisc for which Elliott [4] computed the coefficients of the Faber polynomials of degree $\leqslant 9$. The numbers in Table 6 of the supplement agree with Elliott's except that there is a misprint in his $C_{0}^{(9)}$ and there is an occasional discrepancy of one unit in the last decimal place which he quotes.

Coleman [1] used the Faber polynomials tabulated here, in conjunction with the Lanczos $\tau$-method, to obtain near-minimax polynomial approximations for analytic functions on circular sectors. In that work a Faber polynomial appears as a perturbation term in a differential equation, and consequently the maximum value of the modulus of the polynomial is of interest. In particular, it is useful to have a comparison with the Chebyshev polynomial ( $T$-polynomial) of degree $n$, which is the monic polynomial

$$
T_{n}(z)=z^{n}+a_{n-1} z^{n-1}+a_{n-2} z^{n-2}+\cdots+a_{0}
$$

of smallest maximum modulus on the sector of interest.

An upper bound on the norm of a Faber polynomial,

$$
\left\|\phi_{n}\right\|_{\infty}=\operatorname{Max}_{z \in D}\left|\phi_{n}(z)\right|
$$

where $D=\{z:|z| \leqslant 1,|\arg z| \leqslant \pi / \alpha\}$, with $\alpha \geqslant 2$, may be obtained from the work of Pommerenke [11]. His Satz 3, expressed in our notation, shows that for a convex region of transfinite diameter $\rho$,

$$
\rho^{n} \leqslant\left\|\phi_{n}\right\| \leqslant 2 \rho^{n}
$$

Furthermore, if $\pi \beta$ is the largest exterior angle of the region, then as $n \rightarrow \infty$

$$
\rho^{-n}\left\|\phi_{n}\right\| \rightarrow \beta
$$


for our sector $D, \beta=\max \left\{2\left(1-\alpha^{-1}\right), 3 / 2\right\}$. As $\alpha \rightarrow \infty$, so that the sector becomes the interval $[0,1]$, the upper bound in (24) is attained, as then $\rho=1 / 4$, the Faber polynomial of degree $n$ is a multiple of a shifted Chebyshev polynomial

$$
\phi_{n}(x)=2^{1-2 n} T_{n}^{*}(x),
$$

and $\max _{x \in[0,1]}\left|T_{n}^{*}(x)\right|=1$. The values of $\rho^{-n}\left\|\phi_{n}\right\|$ given in Table 1 show that for each of the sectors considered, this quantity is nearly constant but no obvious pattern is evident in its approach to the limit given by (25).

Table 2, which is based on the work of Grothkopf and Opfer [7], shows $\rho^{-n}\left\|T_{n}\right\|$ for the same sectors, with $n \leqslant 6$; results for $T$-polynomials of higher degree are not available. In the range of this table, $\left\|\phi_{n}\right\| \leqslant 1.5\left\|T_{n}\right\|$. Furthermore, since it is known (Walsh [13, p. 320]) that $\left\|T_{n}\right\| \geqslant \rho^{-n}$, we can conclude from (24) that for all $n$,

$$
\left\|\phi_{n}\right\| \leqslant 2\left\|T_{n}\right\| \text {. }
$$

To use the Faber polynomials in the Lanczos $\tau$-method, it is necessary to have explicit expressions in powers of $z$, as provided by the tables in the supplement.

\section{TABLE 1}

Values of $\left\|\phi_{n}\right\| \rho^{-n}$, the scaled norms of Faber polynomials of degree 1 to 15 , and the limit as $n \rightarrow \infty$. The column headings show the half-angles of the sectors to which they refer.

$\begin{array}{rcccccc}n & 5^{\circ} & 10^{\circ} & 15^{\circ} & 30^{\circ} & 45^{\circ} & 90^{\circ} \\ 1 & 1.890 & 1.784 & 1.681 & 1.389 & 1.347 & 1.392 \\ 2 & 1.994 & 1.977 & 1.949 & 1.813 & 1.617 & 1.532 \\ 3 & 1.902 & 1.826 & 1.766 & 1.648 & 1.575 & 1.469 \\ 4 & 1.979 & 1.925 & 1.855 & 1.622 & 1.442 & 1.479 \\ 5 & 1.920 & 1.876 & 1.844 & 1.702 & 1.497 & 1.507 \\ 6 & 1.960 & 1.884 & 1.811 & 1.669 & 1.535 & 1.486 \\ 7 & 1.937 & 1.903 & 1.852 & 1.644 & 1.500 & 1.490 \\ 8 & 1.945 & 1.874 & 1.827 & 1.681 & 1.486 & 1.503 \\ 9 & 1.949 & 1.899 & 1.828 & 1.673 & 1.505 & 1.492 \\ 10 & 1.938 & 1.886 & 1.844 & 1.652 & 1.515 & 1.494 \\ 11 & 1.952 & 1.886 & 1.824 & 1.672 & 1.493 & 1.502 \\ 12 & 1.938 & 1.896 & 1.836 & 1.674 & 1.497 & 1.494 \\ 13 & 1.949 & 1.882 & 1.837 & 1.658 & 1.508 & 1.496 \\ 14 & 1.942 & 1.893 & 1.826 & 1.667 & 1.506 & 1.501 \\ 15 & 1.944 & 1.888 & 1.839 & 1.674 & 1.492 & 1.496 \\ \infty & 1.944 & 1.889 & 1.833 & 1.667 & 1.500 & 1.500\end{array}$

TABLE 2

Values of $\left\|T_{n}\right\| \rho^{-n}$, the scaled norms of T-polynomials of degree 1 to 6 . The column headings show the half-angles of the sectors to which they refer.

$\begin{array}{cccccc}5^{\circ} & 10^{\circ} & 15^{\circ} & 30^{\circ} & 45^{\circ} & 90^{\circ} \\ 1.734 & 1.576 & 1.464 & 1.301 & 1.331 & 1.299 \\ 1.564 & 1.480 & 1.645 & 1.693 & 1.468 & 1.398 \\ 1.578 & 1.675 & 1.566 & 1.359 & 1.465 & 1.317 \\ 1.677 & 1.486 & 1.357 & 1.322 & 1.268 & 1.220 \\ 1.583 & 1.366 & 1.354 & 1.239 & 1.208 & 1.164 \\ 1.469 & 1.356 & 1.269 & 1.195 & 1.177 & 1.128\end{array}$


However, if numerical values of the Faber polynomials are required, as for example in summing Faber series, that form is not ideal. The tabulated coefficients and the upper bound in (24) show that the norm of a Faber polynomial may be much smaller than the magnitudes of some of its coefficients; significant cancellation would then occur if those coefficients were used to evaluate the polynomial. The alternative is to use the recurrence relation (17), with coefficients $\beta_{k}$ computed as described in Section 3, to generate $\left\{\phi_{n}(z)\right\}$ numerically for each required value of $z$.

Department of Mathematical Sciences

University of Durham

South Road

Durham DH1 3LE, England

1. J. P. Coleman, "Polynomial approximations in the complex plane," J. Comput. Appl. Math. (In press.)

2. J. H. Curtiss, "Faber polynomials and Faber series," Amer. Math. Monthly, v. 78, 1971, pp. $577-596$.

3. S. W. Ellacott, "Computation of Faber series with application to numerical polynomial approximation in the complex plane," Math. Comp., v. 40, 1983, pp. 575-587.

4. G. H. Elliott, The Construction of Chebyshev Approximations in the Complex Plane, Ph.D. Thesis, University of London, 1978.

5. D. Gaier, Vorlesungen über Approximation im Komplexen, Birkhäuser, Basel, 1980.

6. K. O. GedDES \& J. C. MASON, "Polynomial approximation by projection on the unit circle," SIAM J. Numer. Anal., v. 12, 1975, pp. 111-120.

7. U. Grothkopf \& G. OpFer, "Complex Chebyshev polynomials on circular sectors with degree six or less," Math. Comp., v. 39, 1982, pp. 599-615.

8. H. Kober, Dictionary of Conformal Representations, Dover, New York, 1957.

9. J. N. LyNESS \& G. SANDE, "Algorithm 413: Evaluation of normalized Taylor coefficients of an analytic function," Comm. ACM, v. 14, 1971, pp. 669-675.

10. A. I. Markushevich, Theory of Functions of a Complex Variable, Chelsea, New York, 1977.

11. Сh. Pommerenke, "Über die Faberschen Polynome schlichter Funktionen," Math. Z., v. 85, 1964. pp. 197-208.

12. V. I. Smirnov \& N. A. Lebedev, Functions of a Complex Variable, Constructive Theory, Iliffe, London, 1968.

13. J. L. Walsh, Interpolation and Approximation by Rational Functions in the Complex Domain, 5th ed., Amer. Math. Soc. Colloq. Publ., Vol. 20, 1969. 


\section{Supplement to \\ The Faber Polynomials for Circular Sectors}

By John P. Coleman and Russel A. Smith

$$
\begin{aligned}
& \text { Tables of coefficients of Faber polynomials } \\
& \qquad \Phi_{n}(z)=\sum_{k=0}^{n} c_{k}^{(n)} z^{k}
\end{aligned}
$$

for circular sectors. 
$n=1$

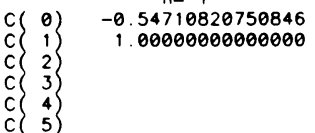

$n=6$

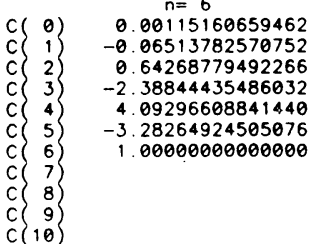

$n=1$

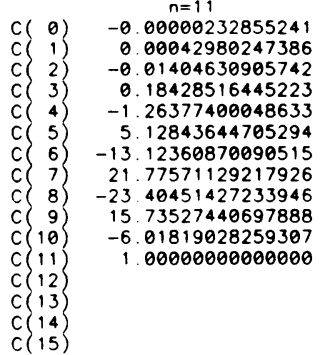

$n=2$
0.16701246657898

$-1.09421641501692$ $n=3$

$-0.04609954204779$ 0.69950978595315

$-1.64132462252538$

1. 00000000000000

$0 \div 8$

$n=7$

$-0.00032944508845$

0.02544447071205

$-0.34119471013575$

1. 76699984979219

$-4.44373688955242$

5. 82277297068105

-3.82975745255922

1.00000000000000

00009571763079 $-0.00954620177328$ 0. 16675290315018 $-1.14254426986018$ 3. 9194866840080 $-7.40922567644968$ 7.8519072436708 $-4.37686566006769$ 1.00000000000000

0. 00000066910250 $-0.0001473233669$ 0.0057093221298 $-0.08919012387962$ 0. 73358679890229 $-3.61080434309925$ 11. 39561761833194 $-23.90135635804360$ 337185971074115 31. 64843279002442 18. $-6.56529849010153$ 100000000000000
$n=13$
0
0. 0000498154226
$-0.0022579505907$
0.04138478286789
$-0.4015906640592$
35191348418737
$-8.94035342328565$
22. 98419458102702
$-40.76354525727285$
4997649206799115
4162136992089810
22.4874894694665
1.0000000000000

$\mathrm{n}=4$

0.01388005930428 $-0.35339015330471$

1. 53133449605045

$-2.18843283003384$

1. 00000000000000

\section{$n=9$}

-0.00002775568542

0.00347373593915

$-0.07654197695835$

0.66849329519433

$-2.98142146292353$

7.57832139709631

$-11.44867518774879$

10. 18036890738373

$-4.92397386757615$

1. 00000000000000

$$
n=14
$$

0.0000000561716

$-0.00001665181861$

- 00087243116326

$-0.01852946197285$

0. 20926779472501

$-1.43576081093505$

6. 45348743834503

$-19.87311469739459$

4298875038196038

$-6596649320570513$

71.47555370762970

$-53.48709013715719$

26. 31258808679502

$-7.65951490511845$

1. 00000000000000 $n=5$

0.00389765187932 0.1579743067106

$-1.07958360017665$

2. 66248659687086

$-2.73554103754230$

100000000000000

$n=10$

0.00000798714894

$-0.00123393522660$

0.0334462177804

$-0.3620033832350$

2. 02812716271008

6. 62083943287081

13. 31127397541997

$-16.72584989564648$

12. 80815796181974

$-5.47108207508461$

1.00000000000000

$$
n=15
$$

- 00000901627021

0. 00000551102608

$-0.0003304020808$

0. 0080460269450

-0. 10455359281036

0. 82963468636079

$-4.34425532062840$

15. 73973721542796 $-4060093918209988$ 75.68247772062159 102. 26239215673389 99. 23153644687584 $-67.4093579109984$

30.43701409484665 $-8.2066231126269$

100000000000000

TABLE 2

COEFFICIENTS OF FABER POLYNOMIALS FOR A SECTOR OF HALF ANGLE 10 DEGREES.

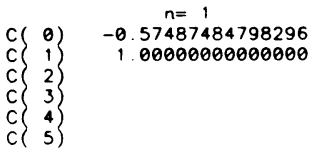

$n=6$

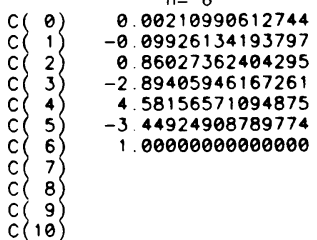

$n=11$

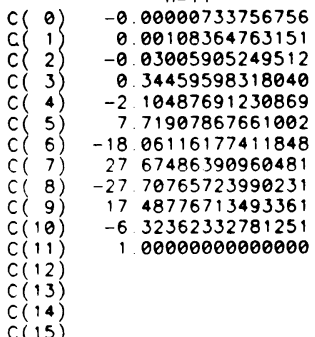

$n=2$

b. 20526420694254 $-1.1497496959659$

$n=7$

$-0.00068654420672$ 0. 04279087619782 $-0.49974331194781$ 2.32127736296250 2. 34095335547540 6. 50184381405887 4.4212393588 $-4.02412393588069$ $n=12$

0. 00000237720618 -0 00041156745676 0. 0134726549378 - 18299776869924 1. 33396005860829 1. 33396005860829 $-5.90347948124036$ 16. 94735444213540 $-3265176590011330$ 42.6759555288193 $-37.39404162261226$ 21. 06045069226086 $-6.89849817579547$

1. 00000000000000

$n=3$

- 0.05109049603599

0.80361794667896

$-1.72462454394887$

I. 00000000000000

$n=8$

0. 00021794177399 $-0.01774784426625$

0. 26788866169690

$-1.63346870098535$

5. 08539559542092

$-8.85577481333991$

8. 75260300801241 -4. 59899878386365

$n=13$

$-0.00000076043088$ 0. 00015427843702 $-0.00588002399896$ 0. 09328232328930 -0. 79857332697095 4. 18551374224731 14. 40436480431009 33.85356599264235 $-55.35416752837731$ 63.03850810489844 $-49.09827990368320$ 24. 96361534043154 -7.47337302377843
1.00000000000000

$$
n=4
$$

(1) 02076322442583

-0. 44411329881276

1. 73245277725879

$-2.29949939193182$

00000000000000

$n=9$

- 0.00007115999598 0.00714295963392

-0.13511934846973 1. 04305377717430 -4 1922942428076 9. 74586211127323 $-1362850910212600$ 11 33384329280938 1. 17387363184660 1. 00000000000000

$n=14$

0.00000024654727

-9.00005718373985

0 00250890015027

$-0.04592954422096$

0. 4556906672940

$-2.78606339405455$

11. 28849225084430 1. 28849225084430 62.81858827075575 $-89.13379129107582$ 89. 90184418472229 $-63.01035734997500$ 29. 19726107944564 $-8.04824787176138$ 1.00000000000008 $n=5$

. 0. 21836834021236 1. 32510786507168 2. 99176869868206 $-2.87437423991478$ .00000000000000

$n=10$

0. 00002277193218 $-0.00280882451516$ 0.06497009663287 $-0.61779843828172$ 3. 10003953254937 -9.19721717438123 17.00512845177949 $-17.00512845177949$ 14.24556466844978 14. 24556466844978 -5.74874847982956
1.00000000000000 $n=15$

00007923425 0. 00002099250023 -0.00104986052680 0.02195130014804

-0. 24960570454680 1. 75802613964680 -8. 26511229519032 2714559850935367 $-6400543021108418$ 109.86222624820260 $-137.59913501476391$ 124. 51450406657632 $-79.32025922834752$ 33. 76138790930317 $-8.62312271974434$ 


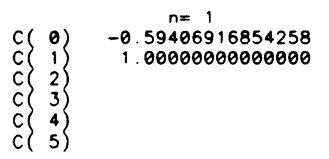

$n=6$

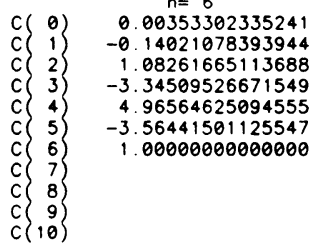

$n=11$

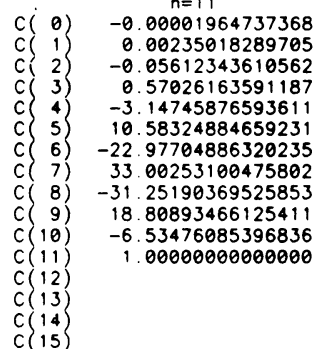

$\begin{aligned} n= & 2 \\ 0 . & 24354270893037\end{aligned}$

$-18813833708516$ $n=3$

$-0.07802203174563$

0.89469132891486

$-1.78220750562774$

.00000000000000 $n=7$

$-0.00127750642102$ 0. 06571312514715 $-0.67796360589007$ 2. 86539243825745 $-6.12120082744717$ 7.02846757898152 $-4.15848417979805$ 1.00000000000000 $n=12$

0. 00600698957833 $-0.00097438193179$ 0.02732696996662 $-0.32735978527466$ 2. 1453684094360 $-8.66065274767496$ 22. 94804986599264 $-41.21933119381668$ 50.6694915573239 $-42.0894386083405$ 22.6363468743544 $-712883002251094$ 1.00000000000000 $n=8$

0.00044542096664 $-0.02967934423999$ o. 39269796222477 $-2.16170842768742$ 6. 19708959555868 $-10.09027664268572$ 9.44420708403037 $-4.75255334834063$ 1.00000000000000 $n=13$

$-0.00000247278584$ 0.00039888037227 $-0.01296665397453$ 0. 18059968924528 $-1.38360088453866$ 6. 58402923831536 20.81415761147054 45. 38251529527867 $-69.44027799724315$ 74.57791863567208 $-55.16823281583736$ 26. 81667726446763 1.00000000000000 $n=4$

0. 02895917861573

0.5331636768463

1. 89875812591220

1. 00000000000000 $n=9$

$-0.00015754674676$ 0.01301870802773 $-0.21441983124714$ 1. 48408141840011 $-5.45381670801380$ 11. 76711467482870 $-15.46198052041273$ 12. 21286476609208 $-5.3466225168832$

1.00000000000000 $n=14$

0. 00000086890895 $-0.00016152448954$

0.00601911821699

$-0.09633639859394$

0.85173342527396 0.85173342527396
-4.70761330223495 $-4.70761330223495$ 17.44469971672226
-45.15846457100129 $-45.15846457100129$ 83. 51776338608219 $-111.23262857888789$ 106.03997498992087 $-70.69794412573066$ 31. 34992583159370 1. 00000000000000 $n=5$

-0.01017591832985

0. 2839069220749

$-1.55230215250907$

3. 25574309992245

$-2.97034584271289$

1. 00000000000000 $n=10$

0.00005619420919 $-0.00558211128981$ o. 11177002804837 $-0.94715541453437$ 4.31848695147800 $-11.81474937211755$ 20. 38942546752172 $-22.44597026860980$ 15. 33444062516666 $-5.94069168542578$ 1.00000000000000 $n=15$

$-0.00000030921185$ 0.00006479423083 $-0.00274178141732$ 0.045 0.04992509530893 . 3. 19562642306497 $-13.68485320281819$ 41. 34078309995714 $-90.41884888760900$ 145.05191219519673 170.95717196766994 46. 49237460985500 36.8882303460020 36. 2360925757326 -8.91103752813868
1.00000000000000 $n=1$

$\left.\begin{array}{l}c \\ c \\ c \\ c \\ c \\ c \\ 1 \\ c \\ c \\ 3 \\ 4 \\ 5\end{array}\right\}$

0.61624027909633

1. 00000000000000 $n=2$

0. 35696695668587

$-1.23248055819265$

1.00000000000000 $n=3$

$-0.14396819888129$

1. 10507855739989

1. 00000000000000 $n=4$
0.06287512285754
-0.78792478503473
2.23294223969462
-2.46496111638530
1.00000000000000

$n=9$
-0.00111511936476
0.05222685612677
-0.59459584632459
3.10734569602923
-9.15139424395587
16.57413342887741
-19.00829540808160
13.56854187487905
-5.54616251186693
1.00000000000000 c( 0) $\quad-0.00021930668382$

c) $13 \quad 0.01436192784051$

c) 2$\} \quad-0.22918035643688$

c) 3 1.69794428696546

c) 4 -7.24452142626779

c) 5 r) $\begin{array}{r}19.69237435777460 \\ -35.86230739037765\end{array}$

c) 7 44 41433715583762

C) 8$)-37.66506378352415$

c) 9 20.76104630001784

c) $\begin{array}{ll}\text { c } & 10 \\ 11 & -6.77864307005959 \\ \text { c } & 1.00000000000000\end{array}$

c $(12)$

C $(13)$

C $\left(\begin{array}{l}14 \\ 15\end{array}\right)$ $n=7$

$-0.00556367359579$

0. 17459489041304

$-1.29926707037639$

4. 33627625663619

7.89504577606312

1.00000000000000 $n=12$

- D. 00009745650633 $-0.00736703704200$ 0.13602909117965 $-1.17055230540288$ 5. 83998841970779 $-18.75976573232356$ 41.01646207825618 67.50223650402107 $-50.36565497039909$ 24. 9269266349583 7. 3948833491559 $n=8$

0.00252412717636 $-0.09670549394607$

0.96285353698961

$-3.83122931193459$

8. 99618920702123

$-12.58408116203256$

10.54191778468073

1. 00000000000000

$n=13$

$-0.00004280821236$

- 00373541285619

$-0.07886952223321$

o. 77835219814176

$-4.47658086531314$

16. 70967879192726

$-42.94828693004128$

78.5089264221238

$-103.49135988326869$

98.15944680983451
-65.62640886169742

29. 47255905147951

$-8.01112362825224$

1.00000000000000 $n=14$

0.00001910352444

$-0.00187504529623$

0.04482610621411

$-0.50197119684237$

3. 28918590018436 $-14.07419841939834$

41.83513775650772 $8953972987699394 \quad 94.59795810735711$ $-162.91853747818740 \quad 238.70136001807591$ $138 \quad 15931710347986-246.72441594344885$ $\begin{array}{llll}-83 & 68134398615986 & 189 & 21940805862394\end{array}$ $34.39794354958142-1047644788725271$ $-8.62736390734856 \quad 39.70308012926404$ $1.00000000000000 \quad-924360418644489$

$$
n=15
$$

- 00000851011536

0.00093331411241

$-0.02504354497935$

0. 31534220059842

-2. 33094495575296

1. 30634801009672 8. 35981101160440 $n=5$

$-0.02927459383730$

-2. 11989584568587

3. 74055800357007

-3.08120139548163
1.00000000000000

$n=10$

0.00048852699560

$-0.0276222914301$

0.37546471651054

$-2.35858098169410$

8. 45543318781628 $-19.03340094890922$ 28.06661137854712
-27.29061677233189 16. 97491804665808 $-6.16240279096326$

100000000000000 1. 00000000000000 
TABLE 5

COEFFICIENTS OF FABER POLYNOMIALS FOR A SECTOR OF HALF ANGLE 45 DEGREES

$n=1$

$\left.\begin{array}{ll}c & 0 \\ c & 1 \\ c & 2 \\ c & 3 \\ c & 4 \\ c & 5 \\ 5\end{array}\right\}$

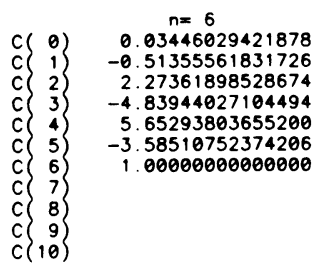

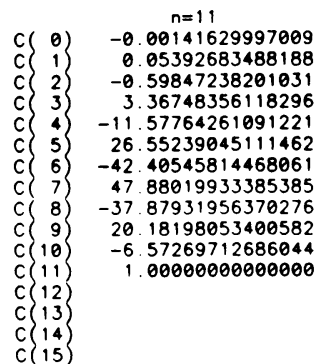

$n=2$

0.45620201698490

$-1.19503584124735$

1.000000000000000 $n=3$

$-0.23308324570747$

1. 21984452367701

$-1.79255376187103$

100000000000000 $n=4$

0. 11476996343846

0.99817570760600

2. 34051469583556

2. 39007168249471

00000000000000 $n=7$

$-0.01788656826698$ 0. 34430264083090
-1.92494840317540 $-1.92494840317540$ $-8.34227322913461$ 7. 84469120510988 $-4.18262544436573$

1.00000000000000 5. 29118819539478

\section{$n=8$ \\ 0.00935345793435 $-0.22332076574083$ 1. 53042742978887 $-5.21822193566418$ 10.47897195410561 $-13.18434720917059$ 10. 39347203913420 \\ i. 00000000000000}

$n=12$

0.00075086371767 $-0.03262650102974$

0. 41298354225828 $-2.65851755126155$ 10.52247837795972 $-28.05743638627881$ 52.87665143273587 52.8766614327358 $-72.04356026480598$ 71.20277386851688 -50.21166191427034
24.15887202989591 24.15887202989591
-7.17021504748412 $-7.17021504748412$ $n=13$

$-0.00040362691494$ 0.01953527471243 $-0.27903527817758$ 2. 03183128797249 -9. 14018666922472 27.90624212015984 60.87925534349887

9754645161744575 1603994742919626 102.05987301209530 $-64.94989742815740$ 28.49279119125244
-7.76773296810779 1.00000000000000 $n=9$

$-0.00506237103358$ o. 14140357979144 $-1.15948081562388$ 4. 76666179818875 $-11.73772134065940$ 18.65489476566494 $-19.57899263942752$ $-5.37766128561309$ 1.00000000000000

\section{$n=14$}

0.00021410937758 $-0.01159730940013$ 0. 18518164127564 $-1.5107703036285$ 7. 64282390267269 $-26.39268287188514$ 65.66420274771792 121. 39827799160778 16940027925361222 1789971284227541 1426.40977467564709
11 $141.90676503837687-266.39773935672713$ $\begin{array}{ll}-82.30735653363858 & 192.32618697505777\end{array}$ 33. $18373801807542-102.49736965898851$ $-8.36525088873147 \quad 38.23171251036484$ $\begin{array}{rr}1.00000000000000 & -8.96276880935515 \\ 1.00000000000000\end{array}$

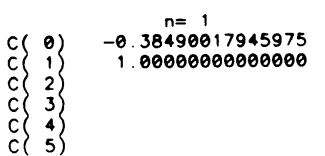

$n=6$

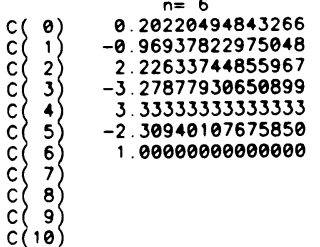

$n=11$

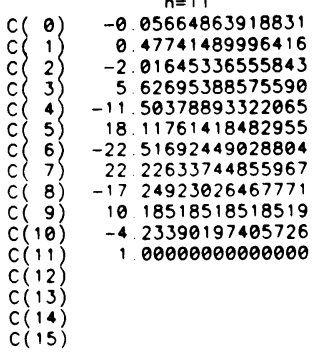

$n=2$

0.51851851851852
-0.76980035891950 1. 00000000000000

$-0.15401482565793$ 0.86241934664431

$-2.33210437952499$

4. 06172839506173

$-4.98944677077454$

4. 40740740740741

$-2.69430125621825$

1. 00000000000000 $n=3$

$-0.48468911487524$

1. 60000000000000

-1.15470053837925
1.00000000000000

\section{$n=8$}

0. 12182349498815

2. 33988721231520

-4. 70116762401868

6. 75445816186557

$-7.18480334991534$

5. 62962962962963

3.07920143567800

1.00000000000000 $n=12$

0. 04281362620447 $-0.40061874199974$

-0.46061874199974
1. 84817589242163

1.84817589242163
-5.63446334048794

-5.63446334048794
12.63667462615794

12.63667462615794
-21.97937833202105

-21.97937833202105
30.47441954986537

30.47441954986537
-34.08029737142384

-34.08029737142384
30.70781893004115

$-21.95356579140799$

12.00000000000000

$-4.61880215351701$

100000000000000 $n=13$

$-0.03282202283009$

๑. 33351323347279

$-1.66979186788285$

552317490105692

$-1347734669432089$

25.63280401775550

$-39.15410421811106$

48. 77295127775238

$-49.76247176142865$

41. 33607681755830

$-27.42770167705778$

13. 96296296296296

$-5.00370233297676$

100000000000000

\section{$n=4$}

- 38134430727023

$-1.08342272736819$

1. 62962962962963

$-1.53960071783900$

1.00000000000000

$n=9$

$-0.09652180941365$ 0. 6556927297668 $-2.27666608207194$ 5. 17421124828532 $-8.49631507251894$ 10.5185185185185 1. -. 92187129274024

-3.46410161513775
1.00000000000000 $n=14$

0. 02554291645948 $-0.27622596446631$ 1. 49042472299394 $-5.31656774494338$ 1401981614348744 28.92532587332406 48.20305734785235 6604388819275667 7498958492099782 $-70.53229790626770$ 54.43484224965706 $-33.7286601704359$ 16.07407407407407 -5. 3886025124365

0000000000000 $n=5$

$-0.06283659387978$ 0.73580857344425

3. 81821253346956

$-2.98758960311838$

$n=10$

0.00270704199080 $-0.08797935198853$ 0. 84578473032532 -4.10282194237149
12.05942474755255 $-23.32709474548254$ 30.76434988822684 27. 73953994818003 $-5.97517920623676$ 1. 00000000000000

$n=15$

$-0.00011266286780$ 0.00683165327009 $-0.12101593577260$ 1. 09713931404376 $-6.18635429573099$ 23.91962153408321 $-67.06389392179792$ $n=5$
-0.27771947105052
1.05624142661180
-1.99577870830982
2.40740740740741
-1.92450089729875
1.00000000000000

$n=10$

0.07474325396352 $-0.56282057548848$ 2. 16357036811236 $-5.47929839377240$ 10. 10618300055886 $-14.23021898952016$ 1558984910836763 $-13.25767284805807$ 13. 25767284805807

8. 51851851851852 $-3.84900179459751$ 1. 00000000000000

$n=15$

-0.01991895613901 o. 22791919247203 $-1.31681813743140$ 5.03828279717029 -14. 27562172334597 31. 74103135521054 $-57.25671634070853$ 85.44776372165490 $-106.60227284003826$ 111.53355687649240 $-97.49378990093458$ 70.34979423868313 $-40.91346352035126$ 18. 33333333333333 $-5.77350269189626$ 1. 00000000000000 